\title{
Acute parotitis in high-dose-rate brachytherapy treatment for skin cancer: a case report
}

\author{
Silvia Rodríguez Villalba, MD, PhDl, Maria José Pérez-Calatayud, PhD², Jose Pérez-Calatayud, PhD13.3 \\ Jose Richart Sancho, MsCl,4, Manvel Santos Ortega, MD, PhDl \\ IRadiotherapy Department, Hospital Clinica Benidorm, Alicante, Spain, ${ }^{2}$ Radiotherapy Department, Hospital General de Valencia, ERESA, \\ Valencia, Spain, ${ }^{3}$ Radiotherapy Department, La Fe University and Polytechnic Hospital, Valencia, Spain, ${ }^{4}$ Radiotherapy Department, Hospital \\ Iniversitario San Juan, San Juan de Alicante, Alicante, Spain
}

J Contemp Brachytherapy 2021; 13, 5: 493-496
DOI: https://doi.org/10.5114/jcb.2021.109994

\section{Purpose}

Brachytherapy (BT) for skin cancer is an accepted alternative technique, where surgical scars may be of cosmetic concern, or for elderly patients due to significant comorbidity. BT is used for macroscopic disease and as an adjuvant to surgery (after local excision due to positive or close margins or other unfavorable prognostic factors) [1-3].

The objective of this case report was to examine clinical evolution of a patient who unexpectedly developed an acute parotitis, after second adjuvant superficial brachytherapy fraction in the preauricular area. This unusual toxicity has been described in patients receiving external radiotherapy (EBRT) whose parotid gland was included within irradiation volume. After ruling out other causes of unilateral parotitis, clinical evolution, and dosimetric analysis, this reaction was attributed to BT treatment, showing the same known clinical situation but rarely appearing with low-dose external beam treatments. To our knowledge, this unusual toxicity has not been published previously, when BT was employed.

\section{Case report}

A 77-year-old patient was referred to our hospital for treatment evaluation in August 2020. His personal history indicated no known drug allergies and type 2 diabetes mellitus. The patient had Bowenoid lesions treated with cryotherapy in the left ciliary area and nasal tip in February 2019. The patient was evaluated by dermatology department of referring hospital, following a diagnosis of erythematous squamous plaque in the left preauricular area, with a maximum diameter of $30 \mathrm{~mm}$, which did not heal following topical treatment of two months duration. A computed tomography (CT) scan was performed and indicated no cervical lymph node involvement. The patient was admitted to plastic surgery department in December 2019 and a surgery was performed to excise the lesion with margins. A histology analysis described the presence of squamous carcinoma, $9.2 \mathrm{~mm}$ thick, moder- ately-differentiated with a $30 \mathrm{~mm}$ maximum diameter on the epidermis. The tumor invaded the reticular dermis and was in contact with the tragus without infiltration (expansive margin). Moreover, it presented focal perineural infiltration (nerve thickness of $0.2 \mathrm{~mm}$ ), with no vascular invasion and free margins (less than $5 \mathrm{~mm}$ ).

After presenting the case to a tumor committee, adjuvant treatment was decided, and BT with plesiotherapy was planned. Our protocol with adjuvant BT includes patients at high-risk of local recurrence, following published guidelines and recommendations [1-3]. In this case, adjuvant treatment was indicated due to very high-risk of recurrence, tumor depth and perineural invasion [4]. A superficial applicator, Freiburg flap (FF) applicator (Nucletron, Elekta AB, Stockholm, Sweden), was used because the treatment area was a flat surface. It is composed of $10 \mathrm{~mm}$ in diameter plastic pellets setting of catheters, $10 \mathrm{~mm}$ apart. Clinical target volume (CTV) was defined with a $10 \mathrm{~mm}$ symmetrical margin on the surface, around surgical bed of the scar, and $5 \mathrm{~mm}$ in depth, as recommended by the ABS and the GEC-ESTRO $[1,2]$.

Computed tomography images were acquired with a $2.5 \mathrm{~mm}$ slice thickness. Treatment plan was to deliver the prescribed dose to CTV up to a minimum total dose of $30 \mathrm{~Gy}$ ( $3 \mathrm{~Gy} / 10$ fractions, twice a day, 6 hours apart for a week from Monday to Friday).

Bolus was not used either above or between the flap and the skin. Bolus above FF is typically not used in this treatment type. Although, calculation algorithm used (TG-43) overestimated the surface dose due to full scatter hypothesis, the use of bolus was not required. This was demonstrated by Vijande et al. [5], who showed that deviations in TG-43-based dose distributions, for a standard FF treatment plan and with respect to Monte Carlo dose distribution, calculated according to composition shape of applicator and surrounding air, were lower than $5 \%$. These results were confirmed by Granero et al. [6], concluding that bolus could just be needed in skin interstitial implants and for cobalt-60 source only. The small
Address for correspondence: Silvia Rodriguez Villalba, MD, Radiotherapy Department, Hospital Clínica Benidorm, Avenida Alfonso Puchades 8, 03501 Benidorm, Alicante, Spain, phone: +34 6065573 46, +34 9660727 25, fax: +34 99660727 24, e-mail: atosrodriguez@gmail.com
Received: 05.04 .2021

Accepted: 10.08 .2021

Published: 14.10 .2021 


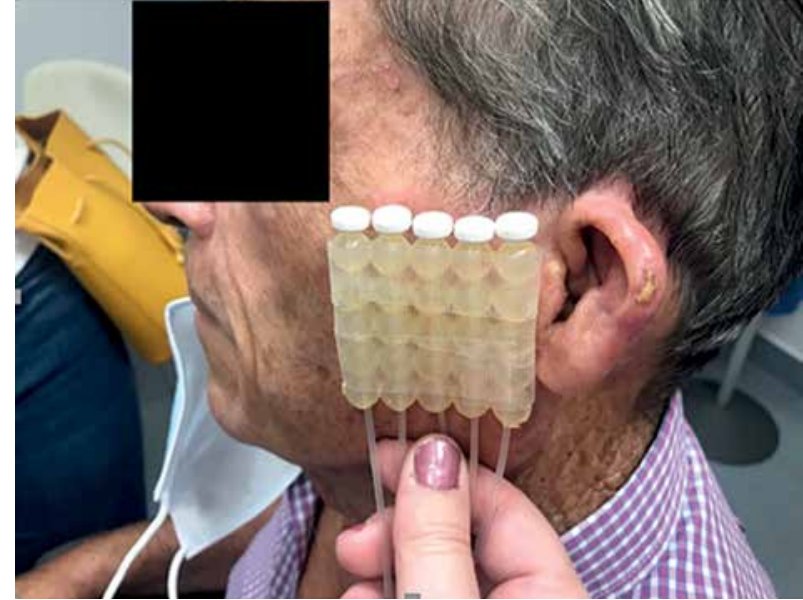

Fig. 1. Simulation of irradiated area with Freiburg flap (FF) applicator

surface underdose due to a scatter defect was compensated by a remarkable gradient between surface dose and prescription dose (5 $\mathrm{mm}$ depth), being approximately $30 \%$. Some hospitals use bolus between FF and the skin to decrease the gradient, and increasing treatment time and peripheral dose.

Our patient started a treatment after complete recovery 10 weeks after the surgery, employing FF with 5 vectors (Figure 1). Four hours after the second session, the patient developed unilateral left acute parotitis with gross facial swelling and pain. Physical examination revealed mild tenderness to palpation over the gland (Figure 2). No systemic symptoms were evident.

Due to this unexpected reaction, BT treatment was discontinued, and parotitis was treated with oral anti-inflammatory drugs. Three weeks later, an ultrasound of the parotid gland and neck was performed, resulting within normal range. Then, it was decided to restart BT treatment, with the remaining 8 fractions of $3 \mathrm{~Gy}$. The patient continued with this procedure, with a full dose administered with the same volume as well as the same applicator, fractionation, and frequency, showing an excellent tolerance.

Acute parotitis did not reappear. For toxicity analyses, common terminology criteria for adverse events
(CTCAE) version 4.0 was employed [7]. The acute skin toxicity was grade 2 (G2), with moderate erythema and patchy moist desquamation, which were resolved 20 days after completing the treatment.

\section{Discussion}

Unilateral acute parotitis is described as a clinical entity attributed to different factors, such as caused by infection, non-infectious systemic illnesses, mechanical obstruction, or medications. Acute viral parotitis begins as a systemic infection located at the parotid gland, resulting in inflammation and swelling of the gland. Acute bacterial parotitis can appear under conditions that predispose to salivary stasis, including dehydration, debilitation, poor oral hygiene, Sjögren syndrome, cystic fibrosis, bulimia/anorexia, ductal stenosis, trauma, immunosuppression, human immunodeficiency virus (HIV), chemotherapy, radiation, malnutrition, or alcoholism. Other causes of unilateral acute mumps are the presence of stones, after anesthesia of a surgical procedure or due to parotid tumors, or metastatic parotid lymph nodes [8]. In the presented case, the patient did not meet any of the above-mentioned criteria. General condition of the patient was excellent, and the tumor removal surgery had been performed 10 weeks before. The patient did not present fever or constitutional syndrome at any time, and the associated pain disappeared within 48 hours after an administration of anti-inflammatory drugs.

Acute parotitis is a secondary effect described already in 1911 [9], with whole brain 2D external radiotherapy treatment, when upper pole of the gland was included in irradiation field during initial fractions.

This acute reaction was also reported by Bergonie et al. [10] and Ceresole [11], involving 10 and 21 cases, respectively, after head and neck irradiation. Kashima et al. [12] in 1965, described this effect in 22 patients who received single-fraction irradiation, consisting of 15.0-27.5 Gy for a variety of malignancies.

This infrequent effect was described in $<5 \%$ of patients at beginning of radiotherapy treatments in areas of the head and neck region [13], during Mantle irradiation [14], whole brain parallel-opposed conformal
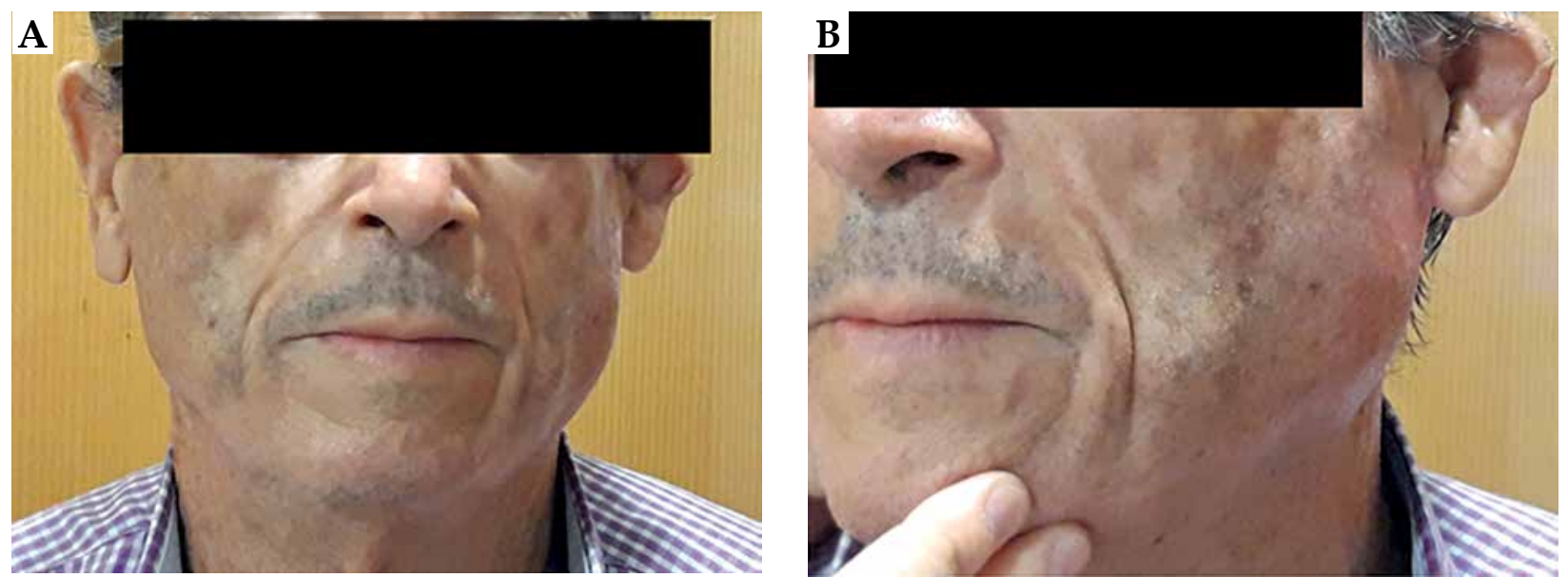

Fig. 2. Patient frontal and lateral view one day after initial two first fractions 


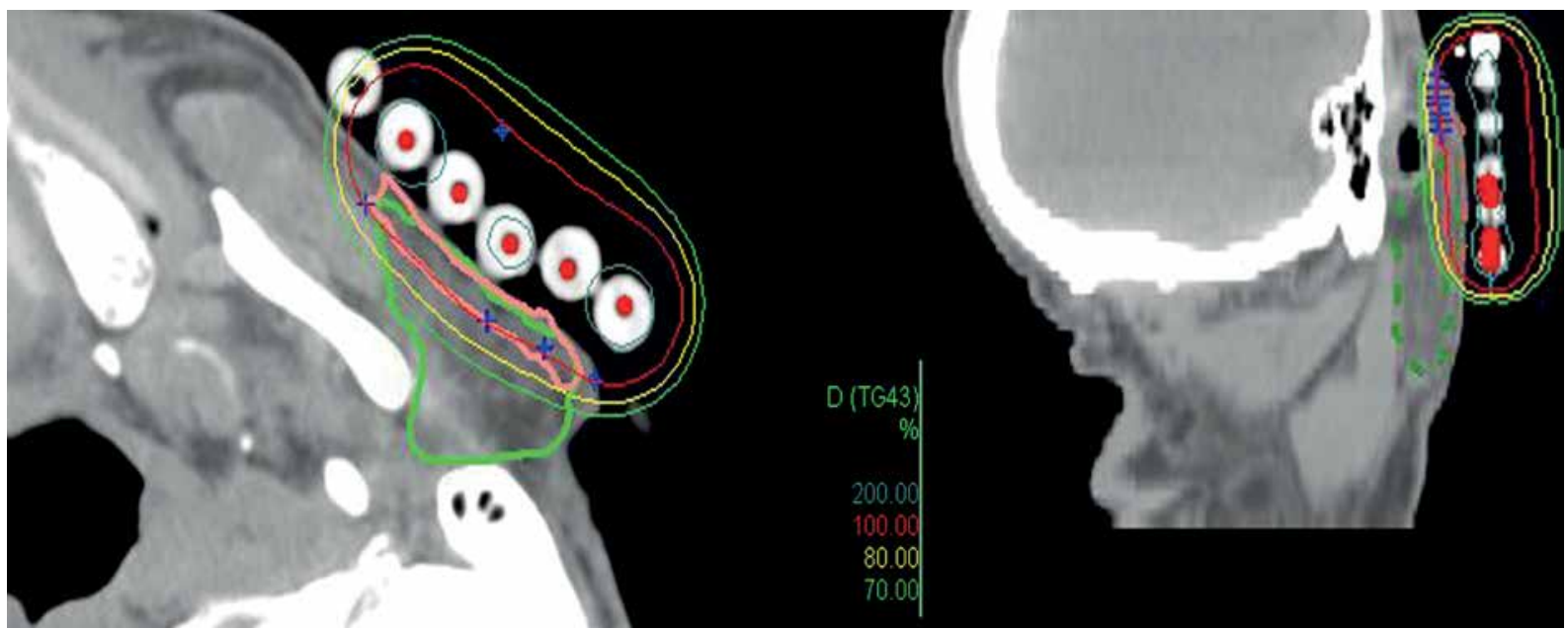

Fig. 3. Dosimetry. Pink line: CTV, green line: parotid gland, red line: $100 \%$ prescription dose, yellow line: $80 \%$ prescription dose, green line: $70 \%$ prescription dose

field treatments [9], and total body irradiation for bonemarrow transplantation [15-17]. Irradiation of salivary glands produces xerostomia, followed by infra-auricular pain and tenderness within 2 to 6 hours post-procedure. The swelling is mostly marked within 12 to 24 hours after irradiation, but rapidly subsides over two to three days. Mild tenderness may persist for up to seven days.

Pathogenesis and morphology of acute parotitis are poorly understood because biopsies are not obtained for obvious reasons. Moreover, etiology of acute parotitis is uncertain, although Kashima et al. [12] noted that 24 hours after irradiation, there was a continued degeneration and loss of acini replacement of acute inflammatory cells by chronic inflammatory cells and fibrosis within lobules. Shannon et al. [18] described a decreased pre-treatment parotid flow after initial treatment with $2.25 \mathrm{~Gy}$, where six of 7 patients experienced no flow following the administration of 2 fractions ( $4.5 \mathrm{~Gy}$ in total).

A close relationship between parotid irradiation and blood amylase levels is well-known [19-21], although individual hypervariability has not been associated with dose [22].

Acute parotitis due to EBRT is described in treatments with partial or total inclusion of the parotid gland. In the presented BT case, the dosimetry was reviewed and the dose in the parotid was evaluated. Dose distribution is presented in Figure 3. To our knowledge, there are no recommended parotid constraints in the published guidelines for skin BT treatments [1-3], nor specific head and neck BT recommendations $[23,24]$. In our patient, $8 \%$ of the volume of the left parotid gland was included in the CTV (Figure 3), receiving a median dose of 1.31 Gy by each fraction and a total of $13.1 \mathrm{~Gy}$. $\mathrm{D}_{2 \mathrm{cc}}$ was $3.03 \mathrm{~Gy}, \mathrm{D}_{\max }$ was $4.30 \mathrm{~Gy}$ per fraction, and 30.3 and $43.0 \mathrm{~Gy}$ in total dose, respectively. The typical EBRT parotid parameter $\mathrm{V}_{30}$ resulted far from the $50 \%$ limit.

The parotitis appears with lower doses, as is described in the literature after EBRT. In our opinion, the novelty of this case reported here was that parotitis occurred during BT treatment, where whole volume of the parotid gland was not included as well as the consideration of the intrinsic gradient of BT itself. Although, from a clinical point of view, this was a self-limiting and clinically manageable event, potentially acute effects in these types of treatments should be considered.

\section{Conclusions}

In this work, acute parotitis in high-dose-rate (HDR) adjuvant superficial skin brachytherapy after a second superficial brachytherapy fraction within the preauricular area is reported. This rare effect can occur in patients treated with EBRT but also with BT, including superficial BT, when parotid is within the field of irradiation.

\section{Disclosure}

The authors report no conflict of interest.

\section{References}

1. Zouhib Z, Kasper M, Pérez-Calatayud J et al. Aspects of dosimetry and clinical practice of skin brachytherapy: The American Brachytherapy Society working group report. Brachytherapy 2015; 14: 840-858.

2. Guinot JL, Rembielak A, Pérez-Calatayud J et al. GECESTRO ACROP recommendations in skin brachytherapy. Radiother Oncol 2018; 126: 377-385.

3. Rodríguez S, Arenas M, Gutiérrez C, et al. Recommendations of the Spanish brachytherapy group (GEB) of Spanish Society of Radiation Oncology (SEOR) and the Spanish Society of Medical Physics (SEFM) for high-dose rate (HDR) non melanoma skin cancer brachytherapy. Practice Guideline Clin Transl Oncol 2018; 20: 431-442.

4. Squamous cell skin cancer. NCCN Clinical Practice Guidelines in Oncology version 1.2021.

5. Vijande J, Ballester F, Ouhib Z et al. Dosimetry comparison between TG-43 and Monte Carlo calculations using the Freiburg flap for skin high-dose-rate brachytherapy. Brachytherapy 2012; 11: 528-535.

6. Granero D, Pérez-Calatayud J, Vijande J et al. Limitations of the TG-43 formalism for skin high-dose-rate brachytherapy dose calculations. Med Phys 2014; 41: 021703.

7. National Institutes of Health. Common Terminology Criteria for Adverse Events (CTCAE). Version 4.03.2010. 
8. Templer J. Parotitis differential diagnoses. Drugs \& Diseases. Otolaryngology and Facial Plastic Surgery. Medscape. Updated: 4 May, 2020.

9. Cairncross JG, Salmon J, Kim JH et al. Acute parotitis and hyperamylasemia following whole-brain radiation therapy. Ann Neurol 1980; 7: 385-387.

10. Bergonie J, Speder F. Sur quelques formes de reactions precoces apres des irradiations Rontgen. Arch Elect Med 1911; 1: 241-245.

11. Ceresole G. A la connaissance des reactions precoces apres des irradiations. Rontgen Arch Elect Med 1912; 20: 304-308.

12. Kashima HK, Lorlja WR, Andrews JR. Postirradiation sialadenitis: A study of the clinical features, histopathologic changes and serum enzyme variations following irradiation of human salivary glands. Am J Roentgen Radium Ther Nucl Med 1965; 94: 271-291.

13. Levendag P, Teguh D, Heijmen B. Textbook. In: Principles and Practice of Radiation Oncology. Halperin E, Pérez C, Brady L. $5^{\text {th }}$ ed. Philadelphia 2008; 913-957.

14. Douglas A, Fein MD, Benjamin W et al. Acute parotitis during mantle irradiation: Incidence, onset, duration, and treatment. Radiat Oncol Invest 1995; 2: 291-294.

15. Tichelli A, Gratwohl A, Speck B et al. Adverse effects of total body irradiation for bone marrow transplantation: prevention and therapy. Experience at Basel July 1979-March 1986. Schweiz Med Wochenschr 1986; 116: 1560-1566.

16. El-Mofty SK. Early membrane injury in lethally irradiated salivary gland cells. Int J Radiat Biol Phys 1981; 39: 55-62.

17. Junglee D. Salivary amylase and pancreatic enzymes in serum after total body irradiation. Clin Chem 1986; 32: 609-610.

18. Shannon IL, Trodahl JN, Starche EN. Radiosensitivity of the human parotid gland. Proc Soc Exp Biol Med 1978; 15: 750-53.

19. Barrett A, Jacobs A, Kohn J et al. Changes in serum amylase and its isoenzymes after whole body irradiation. $\mathrm{Br}$ Med J 1982; 285: 170-171.

20. Becciolini A, Giannardi G, Cionini L et al. Plasma amylase activity as a biochemical indicator of radiation injury to salivary glands. Acta Radiol Oncol 1984; 23: 9-14.

21. Borok TL, Cooper JS. Time course and significance of acute hyperamylasemia in patients receiving fractionated therapeutic radiation to the parotid gland region. Int J Radiat Biol Phys 1982; 8: 1449-1451.

22. Dubray B, Girinski T, Thames H et al. Post-irradiation hyperamylasemia as a biological dosimeter. Radiother Oncol 1992; 24: $21-26$.

23. Bhalavat R, Budrukkar A, Laskar S et al. Brachytherapy in head and neck malignancies: Indian Brachytherapy Society (IBS) recommendations and guidelines. J Contemp Brachytherapy 2020; 12: 501-511.

24. Kovács G, Martinez-Monge R, Budrukkar A et al. GEC-ESTRO ACROP recommendations for head \& neck brachytherapy in squamous cell carcinomas: 1st update - Improvement by cross sectional imaging-based treatment planning and stepping source technology. Radiother Oncol 2017; 122: 248-254. 